\title{
Novel agents for the treatment of childhood leukemia: an update
}

This article was published in the following Dove Press journal:

OncoTargets and Therapy

4 July 2017

Number of times this article has been viewed

\section{Ertugrul Eryılmaz' \\ Cengiz Canpolat ${ }^{2}$}

'Department of Pediatrics, Division of Pediatric Hematology and Oncology, Acibadem Maslak Hospital, ${ }^{2}$ Department of Pediatric Hematology and Oncology, Acibadem Kozyatagi Hospital, Acıbadem University School of Medicine, Istanbul, Turkey
Correspondence: Ertugrul Eryılmaz Department of Pediatrics, Division of Pediatric Hematology and Oncology, Acibadem Maslak Hospital, Acıbadem University School of Medicine, Buyukdere Caddesi No 40, oMaslak 34457, Istanbul, Turkey

Tel +90 2I2304 4197

Fax +90 212 2762291

Email eeryilmaz@acibadem.com.tr

\begin{abstract}
Achieving lower morbidity and higher survival rates in the treatment of childhood leukemia has been a paradigm of success in modern oncology. However, serious long-term health complications occur in very large populations of childhood leukemia survivors, in the case of both acute lymphoid leukemia and acute myeloid leukemia (AML). Additionally, 15\% of acute lymphoid leukemia patients have treatment failures, and rates are even higher in childhood AML. In the last few decades, as a result of well-tested experiments that statistically analyzed treatment cohorts, new agents have emerged as alternatives or supplements to established treatments, in which high survival and/or less morbidity were observed. This review provides an overview of better practice in the treatment of childhood leukemia.
\end{abstract}

Keywords: acute lymphoblastic leukemia, acute myeloid leukemia, pediatric leukemia, systemic therapies

\section{Introduction}

The high success rate of pediatric acute lymphoblastic leukemia (ALL) regimens has been achieved by risk stratification of patients, based on disease features at presentation and responses to induction chemotherapy regimens. Traditional multiagent chemotherapy plays a significant role in the treatment of pediatric ALL and will likely continue to do so for some time. Innovations in cytogenetics and molecular diagnostics are identifying new targets and corresponding therapeutic agents. As novel agents reached developmental milestones and are adopted into practice, higher survival rates and reduced morbidities have been achieved when compared to traditional cytotoxic chemotherapy regimens. Risk feature stratification and intensified chemotherapies for high-risk patients give rise to different treatment patterns from the standard regimens used in both ALL and acute myeloid leukemia (AML).

\section{Relevant milestones in ALL treatment}

In the 1950 s, leukemia patients did not survive for $>2$ years. Improvements in leukemia treatment were guided by lengths of time, with treatment failures. Relevant milestones were met, novel agents flourished in the standard regimens since 1950s and improvements raised survivals every new decade.

In 1948, Farber et al introduced 4-amino-pterylglutamic acid, a folic acid antagonist, that ushered in the chemotherapeutic treatment era for ALL. This agent caused intermittent remission in the leukemic bone marrows; the reads of immature cells in peripheral blood post-mortem studies showed deceleration of the leukemic process, which was not encountered in 200 post-mortem examinations of patients who were not treated with folic acid conjugates. From these data, it is concluded that "transient 
remission" was induced by aminopterin in the treatment of ALL. ${ }^{1}$

The introduction of combination therapies in 1971 overcame resistance to folic acid conjugate therapies. These novel treatment paradigms induced rapid and effective malignant cell lyses. In one study, both sequential use and combination therapies containing antimetabolites with 6-mercaptopurine (6-MP) and methotrexate were analyzed. The use of combination therapies revealed that multimodality treatment maximized remission rates and durations while improving toxicity profiles. ${ }^{2}$

Based on these findings, Pinkel et al proposed a major breakthrough treatment model that still constitutes the backbone of ALL therapy. The treatment flow outlines a multiple-step process as follows: 1) remission induction; 2) intensification/consolidation; 3) continuation and 4) central nervous system (CNS) prophylaxis comprising cranial irradiation and intrathecal methotrexate. ${ }^{3}$

Cranial irradiation, which was essential for CNS prophylaxis, ultimately caused serious sequelae. However, a pediatric oncology group study showed that intrathecal CNS chemotherapeutic prophylaxis added to a systemic chemotherapy backbone was equivalent to cranial irradiation in terms of the length of complete remission (CR). ${ }^{4}$ Subsequently, combined modality treatments using methotrexate, hydrocortisone and cytarabine for CNS prophylaxis increased success rates in both low-risk and high-risk B-cell ALL patients. ${ }^{5}$ Furthermore, addition of dexamethasone further controlled CNS relapse in leukemic patients when compared to prednisolone. ${ }^{6}$

Stratifications based on genetic polymorphisms have identified diverse responses to treatment strategies, leading to patient group-specific treatment modifications. For example, patients with thiopurine S-methyl transferase (TPMT) deficiency develop severe hematopoietic toxicity when treated with standard doses of 6-MP or azathioprine. TPMT has become a standard variant that is tested prior to maintenance treatment. ${ }^{7}$

Recently, significant improvements have been made in the treatment of ALL that have achieved $>90 \%$ 5-year event-free survival for children younger than 14 years. ${ }^{8}$ This success rate has been attributed to: 1) elimination of cranial irradiation; 2) restriction of doxorubicin as an anti-leukemic agent in standard-risk patients, and 3) addition of asparaginase to standard regimens to improve disease-free survival. ${ }^{9}$ Additionally, molecular agents, such as tyrosine kinase inhibitors (TKIs), serine/threonine kinase inhibitors, proteasome inhibitors, epigenetic targets, and antibody-based immunotherapies emerged in the last 2 decades to treat specific target. ${ }^{10}$

\section{New advances in childhood AML therapy}

In pediatric AML, a large number of subtypes have been identified, but most lack targeted therapies to address the mutations; the treatment outcome has improved markedly for children from $20 \%$ in 1980 s to $60 \%$ recently. ${ }^{11}$ Advances have been accomplished through better risk classification, implementation of excellent supportive care measures, and therapeutic adaptations based on patient responses. Additionally, the adaptation of allogeneic hematopoietic stem cell transplantation has raised the cure rates that are regularly measured by the International Acute Myeloid Leukemia Group. ${ }^{12}$

Zavedos $^{\circledR}$ (idarubicin; Pfizer) has activity in AML. This was first discovered in adults. However, by the end of the 20th century, idarubicin was seeing substantial offlabel use in children to avoid daunorubicin (DNR)-induced cardiotoxicity. ${ }^{13}$

Fludara $^{\circledR}$ (fludarabine; Bayer) is a purine analog that interferes with DNA synthesis. Fludarabine produces high response rates in comparison to alkylating agents alone in the treatment of chronic lymphocytic leukemia. Fludarabine was combined with the Idarubicine-Fludarabine, Cytarabine, GranulocyteColony Stimulating Factor (IDA-FLAG) regimen in pediatric patients. In $>50 \%$ of patients, this agent produced CRs lasting for $>12$ months. Thus, fludarabine was recommended as a therapeutic option prior to allogeneic or autologous bone marrow transplantations, particularly as an alternative to standard chemotherapies in certain risk groups. ${ }^{14}$

Remission induction and consolidation regimens have remained unchanged in AML since the 1980s. Overall, pediatric AML cases have been treated as a homogeneous disease, not differentiated by subtypes. One notable exception is the AML M3 subtype, which is also named acute promyelocytic leukemia (APML). The management of APML has changed dramatically following the introduction of the agent Vesanoid $^{\circledR}$ (tretinoin, all-trans retinoic acid [ATRA]; Roche). Leukemic promyelocytes have the unique ability to undergo differentiation with exposure to retinoic acid; both differentiation and apoptosis are stimulated by exposure to arsenic trioxide. ${ }^{15}$ Development of this agent eliminated the need for allogeneic hematopoietic stem cell transplantation in APML patients and led to higher survival rates comparable to low-risk ALL. ${ }^{16}$

\section{Novel agents, in development, that introduce new treatment strategies}

Rates of difficult-to-treat leukemia have declined due to advances in treatment methods and systemic therapy agents. Additionally, strides made in the biology of leukemic cell 
lines have led to earlier recognition of residual diseases. Together, these factors have successfully guided new arms of research and improved the treatment paradigms. Specifically, genetic players in the leukemic DNA landscape have emerged as actionable targets for interventions. The large body of literature that has accumulated recognizing biological underpinnings of childhood leukemia has made tailored therapies both possible and effective. ${ }^{17}$ Relapsed patients, who are rarely cured, will continue to represent the highest unmet need in this space. Although novel therapies are usually developed as single agents, some will also be evaluated in combination with chemotherapeutic backbones. Each class of agent is discussed in detail later.

\section{Targeted therapies and/or agents Enzyme inhibitors}

In Philadelphia chromosome-positive (ph+) ALL, BCR/ ABL1 gene rearrangements lead to actionable protein products. TKIs, eg, Gleevec ${ }^{\circledR}$ (imatinib; Novartis), can specifically inhibit the rearrangement and have improved the outcomes for ALL patients. ${ }^{17}$ It should be noted that two novel agents, Sprycel $^{\circledR}$ (dasatinib; Bristol-Myers Squibb [BMS]) and Tasigna ${ }^{\circledR}$ (nilotinib; Novartis), were developed to overcome the potential over imatinib resistance, after exposure to imatinib. ${ }^{18}$ Both were formerly used successfully to the extent that allogeneic stem cell transplant was deferred in the setting of optimal response to TKI-based regimens in the first CR. ${ }^{19}$

ALL patients with mixed lineage leukemia (MLL) rearrangements have poor treatment outcomes with conventional chemotherapy. MLL rearrangements occur in $80 \%$ of infants and $5 \%$ of older children. FLT3 is one unique gene expression profile that is altered in MLL-rearranged ALL. In all, $18 \%$ of MLL-rearranged ALL cases harbor FLT3-activating mutations that lead to high expression levels. ${ }^{20}$ In vitro studies have demonstrated that a novel enzyme inhibitor of FLT3, Hydrate ${ }^{\circledR}$ (CEP-701, lestaurtinib; Cephalon Inc.), suppresses FLT3-driven leukemic cell survival. ${ }^{21}$ Lestaurtinib is an orally available inhibitor that selectively inhibits FLT3 autophosphorylation, thereby killing leukemic cells. However, lestaurtinib is unlikely to be curative if used as monotherapy in MLL-rearranged ALL patients who overexpress FLT3. ${ }^{22}$

Another actionable mutation in childhood leukemia is purine nucleoside phosphorylase, a catalytic enzyme in the purine salvage pathway. Loss of this enzyme is specifically associated with T-cell lymphopenia and with humoral deficiency. Mundesine ${ }^{\circledR}$ (forodesine; Mundipharma AG) is the most potent agent used to target this pathway. Forodesine is a purine nucleoside phosphorylase inhibitor. In vitro and in vivo studies demonstrate that it targets lymphoid cells and disrupts DNA synthesis. Forodesine has shown promise in early phase clinical trials as a monotherapy for the treatment of relapsed/refractory hematologic malignancies. Trials testing combination therapies are warranted to further improve clinical results. ${ }^{23}$

Other enzyme inhibitors such as the DNA methyltransferase inhibitor Dacogen ${ }^{\circledR}$ (decitabine; Janssen-Cilag International NV/Eisai) and the histone deacetylase inhibitor Zolinza $^{\circledR}$ (vorinostat; Merck/MSD/Taiho) have shown promise as targeted therapies treating relapsed ALL. ${ }^{24}$

Zebularine (CAS 3690-10-6; Sigma-Aldrich) is another potent DNA methyltransferase inhibitor associated with gene demethylation and enhancement of tumor chemosensitivity. Zebularine is a nucleoside analog of cytidine and is considered a prototypical epigenetic therapy for cancer chemoprevention..$^{25}$

\section{Cluster of designation (CD) antibodies}

$\mathrm{CD}$ antibodies are cell surface molecules present on all cells are useful in hematopoietic malignancies because they harbor critical information about lymphoblasts. Evaluation of $\mathrm{CD}$ makeup indicates the type of progenitor cells that are accumulating in bone marrow. Flow cytometry studies are used extensively for both diagnostic and therapeutic purposes in childhood ALL treatment. Minimal residual disease (MRD) sampling at the end of treatment or during follow-up also evaluates $\mathrm{CD}$ status to guide practitioners regarding responses and need for additional treatment modalities. Monoclonal antibodies (mAbs) against CD receptors theoretically serve as targeted therapies to selectively induce blast deformations.

In contrast to current frontline regimens used in adults, $\mathrm{mAb}$ therapies are not extensively used in de novo childhood ALL. One exception is Rituxan ${ }^{\circledR}$ (rituximab; Roche), which is used as the first-line treatment for Burkitt leukemia/ lymphoma ${ }^{26}$ Rituximab targets CD20, a cell surface receptor that is present in approximately half of patients with mature pre-B-cell lymphoma and ALL. As a single agent, rituximab has minimal activity in ALL. In the early 21st century, rituximab added to standard chemotherapy was shown to improve outcomes. Rituximab-based combination therapies have also been promising second-line treatment choices and have been used concomitant within hematopoietic stem-cell transplantation for high-risk patients. ${ }^{27}$

The mAb Campath ${ }^{\circledR}$ (alemtuzumab; Sanofi) targets CD52, which is found on T- and B-lymphocytes, natural killer cells and monocytes. Alemtuzumab was first approved by the US Food and Drug Administration (FDA) in 2001 
for the treatment of B-cell chronic lymphocytic leukemia. ${ }^{28}$ Rituximab and alemtuzumab are examples of naked mAb therapies without an element added to the compound. Another example is LymphoCide ${ }^{\circledR}$ (epratuzumab; Immunomedics), but this agent has shown very limited single-agent activity in pediatric clinical studies. ${ }^{29}$

Other examples of conjugated mAbs includes the antiCD33 antibody Mylotarg ${ }^{\circledR}$ (gemtuzumab ozogamicin; Pfizer/ Wyeth-Ayerst Laboratories), which is a recombinant, humanized anti-CD33 mAb covalently attached to the cytotoxic antitumor antibiotic calicheamicin via a bifunctional linker. Since 2000, when gemtuzumab ozogamicin was approved for clinical use, treatment trials and pilot studies have revealed potential expanded applications along with additional limitations. Therapy can induce blast reduction in childhood AML, which has no further conventional treatment options. ${ }^{30}$

CMC-544 (inotuzumab ozogamicin; Wyeth and UCBCelltech) is a CD22-targeted immunoconjugate of calicheamicin, which was evaluated in Phase III clinical trials in patients with non-Hodgkin's lymphoma, and the research INO-VATE evaluated inotuzumab ozogamicin as part of a chemotherapy regimen in ALL in the frontline setting. ${ }^{31}$

Finally, another antibody-drug conjugate, SGN-CD19A (Seattle Genetics), is composed of a CD19-targeting mAb combined with the cytotoxic agent monomethyl auristatin $\mathrm{F}$. Phase I clinical trials of SGN-CD19A are ongoing in patients with refractory B-lineage ALL. ${ }^{32}$

\section{Immunotherapy}

Recent developments have led to the introduction of several immunotherapy models. Both cell-based therapies and antibodies are being used as targeted therapies. Cell-based therapies can use normal/autologous T-cells collected from the patient that are amplified, activated and targeted against cancer cells. Autologous T-cells are not able to fight a patient's own cancer cells unless modified to gain this activity.

Currently, two cell therapy approaches are under investigation, both of which use autologous T-cell manipulation technologies.

The first is chimeric antigen receptor (CAR) T-cell technology. This is a brand new approach to immunotherapy. This technology involves genetic engineering of autologous T-cells to activate potent cytotoxic cellular effector mechanisms upon recognition of human leukocyte antigen (HLA)independent CD19 antigen-positive ALL cells. The efficacy of this approach was recently demonstrated in clinical trials. CAR expressing T-cells were infused into adult and pediatric patients with B-cell malignancies, where they killed the leukemic cells. ${ }^{33}$ Adult patients in an early phase clinical trial experienced sustained complete responses after treatment with CAR T-cells. This population was refractory to other treatments. Nonetheless, relapsed/refractory ALL patients showed remarkable complete response rates of $\sim 90 \%{ }^{34}$

The second technology utilizes a "smart antibody". Blincyto $^{\circledR}$ (blinatumomab; Amgen) is a novel mAb that combines single-chain antibody fragments of CD19 and CD3. Together these fragments bring a T-cell in close proximity to a malignant lymphoblastic cell, producing redirected lysis. Blinatumomab is infused into the patient where it then engages with normal T-cells and redirects them to tumor cells. As with the CAR T-cell approach, autologous T-cells fight the leukemic cells. This bispecific T-cell-engaging antibody (BiTE) has shown encouraging results in relapsed/ refractory ALL patients and in patients in morphologic remission, but with evidence of minimal residual disease. ${ }^{35}$ In a recent multi-center Phase II trial, blinatumomab was tested in relapsed or refractory B-precursor ALL adult patients. Results showed that blinatumomab was safe and had antileukemic activities. ${ }^{36}$

Given that autologous T-cells are genetically manipulated outside of the body, CAR T-cell technology is an ex vivo process. Conversely, blinatumomab-based BiTE technology works in vivo. CAR T-cells persist in the recipient for months and remain continuously active. In contrast, blinatumomab and other BiTE agents are only active during the course of administration. Overall, both approaches provide proof of principle that patients' autologous T-cells can be manipulated, either in vivo or ex vivo, to act against host leukemic cells. CARs are generated by fusing antigen-binding regions of mAbs or other ligands to membrane-spanning and intracellular-signaling domains. They have recently shown clinical benefit in patients treated with CD19-directed autologous T-cells. These recent successes suggest that modification of T-cells with CARs will be a powerful approach for developing safe and effective cancer therapeutics. ${ }^{37}$

In children, other efforts are being undertaken that test hard-to-treat childhood leukemic cases with immunotherapeutic agents. Autologous tumor cell vaccines are applied de novo shortly after hematopoietic transplant. These personalized tumor vaccines are meant to bolster selective immune responses. The vaccines are composed of the patients' own inactivated leukemic cells combined with an immune stimulant granulocyte-macrophage colony-stimulating factor (GM-CSF). ${ }^{38,39}$

Finally, dendritic cell (DC) vaccinations stimulate immune responses and have demonstrated anti-leukemic effects 
when presenting the Wilms tumor gene (WT1) antigen. Additionally, mRNA-transfected DC vaccines are emerging as nontoxic strategies to prevent or delay relapses in AML. ${ }^{40}$

\section{Radio immunotherapy}

Radio immunotherapy (also called radiolabeled mAb therapy) is another type of conjugated $\mathrm{mAb}$ therapy. The radio immunotherapy mechanism of action links an $\mathrm{mAb}$ to another radioactive isotope, thus delivering radiation directly to cancer cells. Zevalin ${ }^{\circledR}$ (ibritumomab; Spectrum Pharmaceuticals) and iodine I-131-based Bexxar ${ }^{\circledR}$ (tositumomab; GlaxoSmithKline) were both FDA approved in 2002-2003. ${ }^{41}$

Ibritumomab is indicated for adults with previously untreated follicular non-Hodgkin lymphoma (NHL) who achieve partial or complete responses to the first-line chemotherapy. It is also labeled for adults with relapsed or refractory low-grade or follicular B-cell NHL, including those who are refractory to rituximab. ${ }^{42}$

Tositumomab is indicated for relapsed or refractory, low-grade, follicular or transformed NHL, which expresses the CD20 antigen, and for rituximab-refractory NHL. ${ }^{36,43}$ The safety and efficacy of these two agents have not been established in children. ${ }^{42,43}$

\section{Small molecules}

The prototypical examples of effective small molecules for this disease are TKIs such as imatinib, dasatinib and nilotinib. These TKIs target the disease-specific $B C R / A B L 1$ rearranged gene product. ${ }^{18,44}$

Similar research principles are being applied to other mutations and have led to novel agents. The NOTCH1 gene is mutated in approximately half of ALL patients stimulating blastic cells to proliferate. Several drugs are in development to block activating NOTCH1 mutation, thereby preventing the leukemia cells from dividing. ${ }^{45}$

Researchers have identified several other targetable mutated gene products. These can target the gene, which is involved in methylation, as well as multiple targets, inhibiting Janus kinase (JAK), signal transducer and activator of transcription (STAT), mammalian target of rapamycin (mTOR) plus the phosphatidylinositol-3-kinase (PI3K) pathways. ${ }^{45-49}$

\section{Non-targeted therapies and/or agents Chemotherapy}

New chemotherapy drugs with considerable efficacy are approved by the FDA for treatment of ALL. For example,
Evoltra $^{\circledR}$ and Clolar ${ }^{\circledR}$ (clofarabine; Genzyme [US]/Bioenvision [EU]) was the first agent approved for relapsed ALL in children and young adults that raised complete cure rates from $20 \%$ to $30 \%$. Additionally, Arranon ${ }^{\circledR}$ (nelarabine; Novartis) was approved for T-cell ALL in both adults and children, which boasts a single-agent CR rate of $\sim 30 \% .{ }^{50}$

Both clofarabine and nelarabine are being tested as frontline treatment in combination with other chemotherapy regimens. Clofarabine is being studied in pediatric patients, while nelarabine has been also focused on adults with T-cell ALL. ${ }^{51}$

A third drug that was recently approved for ALL is Erwinaze $^{\circledR}$ (asparaginase Erwinia chrysanthemi; Jazz Pharmaceuticals/Ohara Pharmaceutical). Asparaginase Erwinia chrysanthemi is indicated for patients who develop hypersensitivity to Escherichia coli-derived asparaginase and achieve efficacious therapeutic serum drug concentrations. ${ }^{52}$

\section{De novo liposomal agents}

Liposomes are organic structures of lipid molecules that form hollow spheres that encapsulate a wide range of cargo molecules. During the last several decades, liposomes have developed as extremely flexible vehicles for delivering chemotherapies to cancer cells, while minimizing unwanted systemic side effects. Marqibo ${ }^{\circledR}$ (liposomal vincristine sulfate; Talon Therapeutics), DepoCyte ${ }^{\circledR}$ (sustained-release DepoFoam cytarabine; Mundipharma International), DaunoXome ${ }^{\circledR}$ (liposomal formulation DNR; Galen/Sayre Therapeutics), Myocet $^{\circledR}$ (liposomal formation doxorubicin; Cephalon/Teva Pharmaceuticals) and Oncaspar ${ }^{\circledR}$ (pegaspargase, PEG-L-asparaginase; Shire/Medac/Rhone-Poulenc) are novel liposomal agents that are essential to standard childhood leukemia treatment regimens.

Liposomal vincristine sulfate is a recently approved liposomal form of vincristine. The molecular structure of liposomal vincristine sulfate is a sphingomyelin- and cholesterol-based liposome that encapsulates vincristine. The agent is delivered in 1 hour weekly infusions. In adult $\mathrm{Ph}$-negative ALL patients, vincristine is slowly released from the liposome and delivered into the tissues more efficiently than with the standard preparation. ${ }^{53}$ Trials are ongoing, testing liposomal vincristine sulfate in pediatric patients. For example, the pivotal trial, "Vincristine Sulfate Liposome Injection Marqibo ${ }^{\circledR}$ In Combination with UK ALL R3 Induction Chemotherapy for Children, Adolescents, and Young Adults with Relapsed ALL" is currently recruiting patients in the UK and is sponsored by the Therapeutic Advances in Childhood Leukemia Consortium (NCT 02879643). 
Sustained-release DepoFoam cytarabine is a slow-release formulation of cytarabine that is considered a safe and efficient component of triplet intrathecal CNS prophylaxis regimens. The use of sustained-release DepoFoam cytarabine, once every 2 weeks, maintains cytotoxic concentrations of cytarabine in the cerebrospinal fluid for $>14$ days. Standard cytarabine injections must be performed twice weekly. ${ }^{54}$

Liposomal formulation DNR is a liposome encapsulated form of DNR. It has better pharmacokinetics and pharmacodynamics and improved cardiotoxicity compared to free-form DNR. The use of liposomal formulation DNR improved early treatment responses in relapsed AML in children. ${ }^{55,56}$

Liposomal formation doxorubicin is a non-pegylated liposomal doxorubicin with an impressive safety profile, particularly regarding acute cardiac toxicity, in childhood leukemia. However, special attention must be given to control infectious complications, as both liposomal formation doxorubicin and liposomal formulation DNR may easily cause severe myelosuppression. ${ }^{57}$

Pegaspargase is a pegylated formula of L-asparaginase that is used to replace the native form of the molecule. Replacement with a pegylated version decreases immunogenicity and increases circulating half-life. PEG-L-asparaginase is well-tolerated and can be utilized in patients who are hypersensitive to un-pegylated products. ${ }^{58}$

Annamycin $^{\circledR}$ (L-annamycin; Moleculin Biotech) is a liposomal formulation that has promising activities in both the pediatric and adult leukemic settings. This agent is intended for the treatment of relapsed or refractory AML. Currently, L-annamycin has been tested in six early phase clinical trials spanning 114 patients. There have been no reports of cardiotoxicity. Two of the clinical trials focused on leukemia. Data from these trials indicated that L-annamycin had less dose-limiting toxicity when compared to patients receiving doxorubicin. Additionally, cardiotoxicity was eliminated while avoiding multi-drug resistance effects. ${ }^{59}$

\section{Interferons and interleukins}

Interferons and interleukins are naturally secreted by various types of cells in the body. They act on other cells to stimulate or inhibit certain actions of the cells. For example, some interferons serve as cytokines that increase production of immune cells as part of a natural response to infection and cancer. Interferons and interleukins are sometimes referred to as nonspecific immunotherapies. For example, interferon- $\alpha$ molecules are produced by lymphocytes to resist infections and cancers. High-dose interferon- $\alpha$ behaves like a chemotherapeutic agent by blocking cancer cell growth. Interferons also stimulate innate immune-based defenses. Using interferon- $\alpha$ with other immune modifiers augments the effects of chemotherapy. Interferon- $\alpha$ molecules are administered either daily or multiple times per week. They are commonly used to treat patients with hairy cell leukemia, chronic myeloid leukemia, NHL and cutaneous T-cell lymphoma.$^{60}$ Novel formulations of interferon such as Pegasys ${ }^{\circledR}$ (pegylated-IFN- $\alpha$; Roche/Chugai) are available on once weekly dosing schedules. ${ }^{61}$

\section{Donor lymphocyte infusion (DLI)}

DLI is another type of immunotherapy. First, patients receive an allogeneic stem cell transplant from a donor. Next, lymphocytes are collected from the same donor and then infused into the patient. The donor and the recipient may have very similar, but not necessarily identical, blood types. As a result, the donor lymphocytes may identify recipient cells as targets. Infusing allografts of Granulocyte-Colony Stimulating Factor (GCSF)-mobilized peripheral blood progenitor cells, allodepleted donor T-cells and infusions of donor-derived, ex vivo-expanded CD8(+) cytotoxic T lymphocytes as DLI have decreased relapse rates and improved transplant outcomes. ${ }^{62}$

\section{Discussion}

New modalities used as best treatment practice for both ALL and AML are remarkable in their diversity. Over the last decade, important biological and clinical differences have been identified between leukemic cells at diagnosis and relapse. For example, cancers may acquire new chromosomal abnormalities and gene mutations and have reduced responsiveness to chemotherapeutic agents. Nonetheless, the clinical relevance of new agents and the best settings in which to apply these agents might take some time to elucidate, given that many trials share common methods and designs. However, high-risk patients who benefited from recent trials experienced higher survival rates and the same or less morbidity, suggesting that these agents will be added to the pediatric leukemia arsenal.

Overall, outcomes for patients with pediatric hematopoietic malignancies are excellent. Thus, new trials to evaluate novel therapies in children and young adults with refractory, resistant or relapsed disease have a high bar to pass and will be a challenge. For these rare malignancies, small sample sizes take a long time to analyze, because they are reached slowly in this setting. Given the fragility of risk populations and the success of current agents, combination therapies may be difficult to test. Nonetheless, they are probably necessary 
to further improve outcomes in the relapsed or resistant population. Phase II window studies could partly overcome this problem, as could Phase II multiarm studies. ${ }^{63}$

Owing to very high cure rates in childhood ALL, current clinical research goals may be non-inferiority or reduced toxicity. However, in the relapsed or resistant setting, evidence of better survival with acceptable toxicity may be the aim. Describing the safety and efficacies of new therapies or agents, which are then translated into improved patient outcomes, will face many challenges. Collaborative relationships between scientists, academics, clinical investigators and regulatory bodies should shorten the developmental time frame needed for approval of pediatric hematologic malignancy treatments.

\section{Summary}

The arsenal of agents in the fight against childhood leukemia is expanding. Novel agents hold the promise of being used as single agents or being combined with chemotherapy backbones.

In the near future, personalized treatment strategies will be developed based on detailed genetic and epigenetic characteristics or responses to early treatments and MRD monitoring. Such strategies will require precise supportive care. There are many questions that need to be addressed to determine whether treatment should be limited to patients with overt disease or whether it should include those with MRD. Future studies will resolve whether novel agents that have limited activities are improved when combined with chemotherapy. The most effective types of chemotherapy used in combination therapies remain to be determined and will be selected based on better outcomes and less toxicity.

\section{Disclosure}

The authors report no conflicts of interest in this work.

\section{References}

1. Farber S, Diamond LK, Mercer RD, et al. Temporary remissions in acute leukemia in children produced by folic acid antagonist,4-aminopteroylglutamic acid. N Engl J Med. 1948;238(23):787-793.

2. Frei E, Freireich EJ, Gehan E, et al. Studies of sequential and combination antimetabolite therapy in acute leukemia: 6-mercaptopurine and methotrexate. Blood. 1961;18:431-454.

3. Pinkel D. Five-year follow-up of "total therapy" of childhood lymphocytic leukemia. JAMA. 1971;216:648-652.

4. Sullivan MP, Chen T, Dyment PG, Hvizdala E, Steuber CP. Equivalence of intrathecal chemotherapy and radiotherapy as central nervous system prophylaxis in children with acute lymphatic leukemia: a pediatric oncology group study. Blood. 1982;60(4):948-958.

5. Pullen J, Boyett J, Shuster J, et al. Extended triple intrathecal chemotherapy trial for prevention of CNS relapse in good-risk and poor-risk patients with B-progenitor acute lymphoblastic leukemia: a Pediatric Oncology Group study. J Clin Oncol. 1993;11(5):839-849.
6. Jones B, Freeman AI, Shuster JJ, et al. Lower incidence of meningeal leukemia when prednisone is replaced by dexamethasone in the treatment of acute lymphocytic leukemia. Med Pediatr Oncol. 1991;19(4): 269-275.

7. Krynetski EY, Schuetz JD, Galpin AJ, Pui CH, Relling MV, Evans WE. A single point mutation leading to loss of catalytic activity in human thiopurine S-methyltransferase. Proc Natl Acad Sci US A. 1995;92(4): 949-953.

8. Murphy SL, Xu J, Kochanek KD. Deaths: Final Data for 2010. National Vital Statistics Reports. 61(4). Hyattsville, MD: National Center for Health Statistics; 2013.

9. Sallan SE, Hitchcock-Bryan S, Gelber R, Cassady JR, Frei E 3rd, Nathan DG. Influence of intensive asparaginase in the treatment of childhood non-T-cell acute lymphoblastic leukemia. Cancer Res. 1983; 43(11):5601-5607.

10. Brown P, Hunger SP, Smith FO, Carroll WL, Reaman GH. Novel targeted drug therapies for the treatment of childhood acute leukemia. Expert Rev Hematol. 2009;2(9):145.

11. Rubnitz JE, Inaba $H$, Leung WH, et al. Definition of cure in childhood acute myeloid leukemia. Cancer. 2014;120(16):2490-2496.

12. Rubnitz JE. How I treat pediatric acute myeloid leukemia. Blood. 2012;119(25):5980-5988.

13. Ritter J, Creutzig U, Zimmerman M. Specific role of idarubicin during induction therapy of childhood acute myeloblastic leukemia acute leukemia VI. Ser Haematol Blood Transfus. 1997;38:396-399.

14. Fleischhack G, Hasan C, Graf N, Mann G, Bode U. IDA-FLAG (idarubicin, fludarabine, cytarabine, G-CSF), an effective remission-induction therapy for poor-prognosis AML of childhood prior to allogeneic or autologous bone marrow transplantation: experiences of a phase II trial. Br J Haematol. 1998;102(3):647-655.

15. Huang M, Yu-Chen Y, Shu-Rong C, et al. Use of all-trans retinoic acid in the treatment of acute promyelocytic leukemia. Blood. 1988;72: $567-578$.

16. Tallman MS, Altman JK. How I treat acute promyelocytic leukemia. Blood. 2009;114(25):5126-5135.

17. Evans WE, Relling MV, Rodman JH, Crom WR, Boyett JM, Pui CH Conventional compared with individualized chemotherapy for childhood acute lymphoblastic leukemia. N Engl J Med. 1998;338(8):499-505.

18. Schultz KR. Improved early event-free survival with imatinib in Philadelphia chromosome-positive acute lymphoblastic leukemia: a Children's Oncology Group Study. J Clin Oncol. 2009;27(31):5175-5181.

19. Steinberg M. Dasatinib: a tyrosine kinase inhibitor for the treatment of chronic myelogenous leukemia and Philadelphia chromosome-positive acute lymphoblastic leukemia. Clin Ther. 2007;29(11):2289-2308.

20. Small D. Targeting FLT3 for treatment of leukemia. Semin Hematol. 2008;45(3 suppl 2):S17-S21.

21. Fathi A, Levis M. FLT3 inhibitors: a story of the old and the new. Curr Opin Hematol. 2011;18(2):71-76.

22. Brown P, Levis M, McIntyre E, Griesemer M, Small D. Combinations of the FLT3 inhibitor CEP-701 and chemotherapy synergistically kill infant and childhood MLL-rearranged ALL cells in a sequencedependent manner. Leukemia. 2006;20(8):1368-1376.

23. Al-kali A, Gandhi V, Ayoubi M, Keating M, Ravandi F. Forodesine: review of preclinical and clinical data. Future Oncol. 2010;6(8): 1211-1217.

24. Burke MJ, Lamba JK, Pounds S, et al. A therapeutic trial of decitabine and vorinostat in combination with chemotherapy for relapsed/ refractory acute lymphoblastic leukemia (ALL). Am J Hematol. 2014; 89(9):889-895.

25. Andrade AF, Borges KS, Castro-Gamero AM, et al. Zebularine induces chemosensitization to methotrexate and efficiently decreases AhR gene methylation in childhood acute lymphoblastic leukemia cells. Anticancer Drugs. 2014;25(1):72-81.

26. Leone G, Sica S, Voso MT, Rutella S, Pagano L. Treatment of acute leukaemias with monoclonal antibodies: current status and future prospects. Cardiovasc Hematol Agents Med Chem. 2006;4(1):33-52. 
27. Bhojwani D, Pui CH. Relapsed childhood acute lymphoblastic leukaemia. Lancet Oncol. 2013;14(6):e205-e217.

28. Dyer MJ, Kelsey SM, Mackay HJ, et al. In vivo 'purging' of residual disease in CLL with Campath-1H. Br J Haematol. 1997;97(3): 669-672.

29. Raetz EA, Cairo MS, Borowitz MJ, et al. Re-induction chemoimmunotherapy with epratuzumab in relapsed acute lymphoblastic leukemia (ALL): phase II results from Children's Oncology Group (COG) study ADVL04P2. Pediatr Blood Cancer. 2015;62(7):1171-1175.

30. Linenberger ML. CD33-directed therapy with gemtuzumab ozogamicin in acute myeloid leukemia: progress in understanding cytotoxicity and potential mechanisms of drug resistance. Leukemia. 2005;19(2): $176-182$.

31. Thomas X. Profile of inotuzumab ozogamicin and its potential in the treatment of acute lymphoblastic leukemia. Blood Lymphatic Cancer. 2014;4:1-8

32. Albertson TM, Sandalic L, Baiteng Z. A novel Anti-CD19 antibody drug conjugate. Cancer Res. 2014;74(19):DDT01-04.

33. Shi H, Sun M, Liu L, Wang Z. Chimeric antigen receptor for adoptive immunotherapy of cancer: latest research and future prospects. $\mathrm{Mol}$ Cancer. 2014;13:219.

34. Maus MV, June CH. Making better chimeric antigen receptors for adoptive T-cell therapy. Clin Cancer Res. 2016;22(8):1875-1884.

35. Hoffman LM, Gore L. Blinatumomab, a bi-specific anti-CD19/ CD3 BiTE $(\mathbb{R})$ antibody for the treatment of acute lymphoblastic leukemia: perspectives and current pediatric applications. Front Oncol. 2014;4:63

36. Topp MS, Gokbuget N, Stein AS, et al. Safety and activity of blinatumomab for adult patients with relapsed or refractory B-precursor acute lymphoblastic leukaemia: a multicentre, single-arm, phase 2 study. Lancet Oncol. 2015;16(1):57-66.

37. Dai H, Wang Y, Lu X. Chimeric antigen receptors modified T-cells for cancer therapy. J Natl Cancer Inst. 2016;108(7):djv439.

38. Srivatsan S, Patel JM, Bozeman EN, et al. Allogeneic tumor cell vaccines. Hum Vaccin Immunother. 2014;10(1):52-63.

39. Shah NN, Dave H, Wayne AS. Immunotherapy for pediatric leukemia. Front Oncol. 2013;3:166.

40. Berneman ZN, Velde AV, Anguille S, et al. Prevention of relapse in acute myeloid leukemia by dendritic cell vaccination: report on a Phase II Study with 29 patients. Blood. 2013;122:236.

41. Oriuchi N, Higuchi T, Hanaoka H, Iida Y, Endo K. Current status of cancer therapy with radiolabeled monoclonal antibody. Ann Nucl Med. 2005;19(5):355-365.

42. Wiseman GA, White CA, Witzig TE. Radioimmunotherapy of relapsed non-Hodgkin's lymphoma with zevalin, a 90Y-labeled anti-CD20 monoclonal antibody. Clin Cancer Res. 1999;5:3281s-3286s.

43. Rao AV, Akabani G, Rizzieri DA. Radioimmunotherapy for nonHodgkin's lymphoma. Clin Med Res. 2005;3(3):157-165.

44. Bernt KM, Hunger SP. Current concepts in pediatric Philadelphia chromosome-positive acute lymphoblastic leukemia. Front Oncol. 2014;4:54.

45. Ye Q, Jiang J, Guangun Z, et al. Small molecule activation of NOTCH signaling inhibits acute myeloid leukemia. Sci Rep. 2016;6:26510.

46. Borkin D, Pollock J, Kempinska K, et al. Property focused structurebased optimization of small molecule inhibitors of the protein-protein interaction between menin and mixed lineage leukemia (MLL). J Med Chem. 2016;59(3):892-913.

OncoTargets and Therapy

\section{Publish your work in this journal}

OncoTargets and Therapy is an international, peer-reviewed, open access journal focusing on the pathological basis of all cancers, potential targets for therapy and treatment protocols employed to improve the management of cancer patients. The journal also focuses on the impact of management programs and new therapeutic agents and protocols on
47. Kim BH, Yin $\mathrm{CH}$, Guo Q, et al. A small-molecule compound identified through a cell-based screening inhibits JAK/STAT pathway signaling in human cancer cells. Mol Cancer Ther. 2008;7(9):2672-2680.

48. Coffey RT, Shi Y, Long MJ, Marr MT, Hedstrom L. Ubiquilin mediated small molecule inhibition of mammalian target of rapamycin complex 1 (mTORC1) signaling. J Biol Chem. 2016;291(10):5221-5233.

49. Martelli AM, Yakern MN, Tabellini G, et al. Phosphoinositide 3-kinase/ Akt signaling pathway and its therapeutical implications for human acute myeloid leukemia. Leukemia. 2006;20(6):911-928.

50. Fullmer A, O’Brien S, Kantarjian H, Jabbour E. Novel therapies for relapsed acute lymphoblastic leukemia. Curr Hematol Malig Rep. 2009;4(3):148-156.

51. DeAngelo DJ. Nelarabine for the treatment of patients with relapsed or refractory T-cell acute lymphoblastic leukemia or lymphoblastic lymphoma. Hematol Oncol Clin North Am. 2009;23(5):1121-1135.

52. Fernandez CA, Stewart E, Panetta JC, et al. Successful challenges using native $E$. coli asparaginase after hypersensitivity reactions to PEGylated E. coli asparaginase. Cancer Chemother Pharmacol. 2014; 73(6):1307-1313.

53. Davis T, Farag SS. Treating relapsed or refractory Philadelphia chromosome-negative acute lymphoblastic leukemia: liposomeencapsulated vincristine. Int J Nanomedicine. 2013;8:3479-3488.

54. Glantz MJ, LaFolette S, Jaeckle KA, et al. Randomized trial of a slowrelease versus a standard formulation of cytarabine for the intrathecal treatment of lymphomatous meningitis. J Clin Oncol. 1999;17(10): 3110-3116.

55. Sedki M, Vannier JP, Leverger G, et al. Liposomal daunorubicin (Daunoxome) and polyethylated glycol conjugated asparaginase (PEGASPA) in children with relapsed and refractory acute lymphoblastic leukemia treated on compassionate basis. J Egypt Natl Canc Inst. 2008; 20(1):55-62.

56. Kaspers GJ, Zimmermann M, Reinhardt D, et al. Improved outcome in pediatric relapsed acute myeloid leukemia: results of a randomized trial on liposomal daunorubicin by the International BFM Study Group. J Clin Oncol. 2013;31(5):599-607.

57. Rafiyath SM, Rasul M, Lee B, Wei G, Lamba G, Liu D. Comparison of safety and toxicity of liposomal doxorubicin vs. conventional anthracyclines: a meta-analysis. Exp Hematol Oncol. 2012;1(1):10.

58. Kurtzberg J. Polyethylene Glycol-conjugated L-asparaginase versus native L-asparaginase in combination with standard agents for children with acute lymphoblastic leukemia in second bone marrow relapse: a Children's Oncology Group Study. J Pediatr Hematol Oncol. 2011; 33(8):610-616.

59. Sabnis N, Bowman WP, Lacko AG. Lipoprotein based drug delivery: potential for pediatric cancer applications. World J Pharmacol. 2015; 4(2):172-179.

60. Velders MP. Prospects for immunotherapy of acute lymphoblastic leukemia. Nature. 2001;15(5):701-706.

61. Preudhomme C, Guilhot J, Nicolini FE, et al. Imatinib plus peginterferon alfa-2a in chronic myeloid leukemia. $N$ Engl J Med. 2010; 363(26):2511-2521.

62. Chang YJ, Huang XJ. Donor lymphocyte infusions for relapse after allogeneic transplantation: when, if and for whom? Blood Rev. 2013; 27(1):55-62.

63. Thomas A. How can we improve on the already impressive results in pediatric ALL? Hematology Am Soc Hematol Educ Program. 2015; 2015(1):414-419.

\section{Dovepress}

patient perspectives such as quality of life, adherence and satisfaction. The manuscript management system is completely online and includes a very quick and fair peer-review system, which is all easy to use. Visit http://www.dovepress.com/testimonials.php to read real quotes from published authors. 